\title{
Proretinal nanoparticles: stability, release, efficacy, and irritation
}

Pimolphan Pisetpackdeekul,' Piyapan Supmuang, ${ }^{2}$ Porntip Pan-In, ${ }^{3}$ Wijit Banlunara, ${ }^{4, *}$ Benchaphorn Limcharoen, ${ }^{4}$ Chayada Kokpol, ${ }^{5, *}$ Supason Wanichwecharungruang ${ }^{3,6, *}$

'Program in Technopreneurship and Innovation Management, Graduate School, ${ }^{2}$ Program in Biotechnology, ${ }^{3}$ Department of Chemistry, Faculty of Science, ${ }^{4}$ Department of Pathology, Faculty of Veterinary Science, Chulalongkorn University, ${ }^{5}$ Division of Dermatology, Department of Medicine, Faculty of Medicine, Ramathibodi Hospital, ${ }^{6}$ Nanotec-Chulalongkorn University Center of Excellence on Food and Agriculture,

Chulalongkorn University, Bangkok, Thailand

*These authors contributed equally to this work

\section{Video abstract}

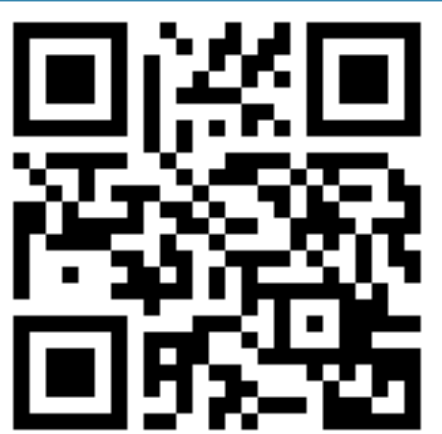

Point your SmartPhone at the code above. If you have a QR code reader the video abstract will appear. Or use: http://youtu.be/EoDWfm0tiBO

Correspondence: Supason Wanichwecharungruang Department of Chemistry, Faculty of Science, Chulalongkorn University, Phayathai Road, Bangkok 10330, Thailand

Tel +6622187634

Fax +6622541309

Email psupason@chula.ac.th
This article was published in the following Dove Press journal:

International Journal of Nanomedicine

21 July 2016

Number of times this article has been viewed

Abstract: Despite many potent biological activities, retinoids such as retinoic acid (RA) and retinal possess dose-related broad side effects. In this study, we show that this problem, which has been unsolvable for a long time, can be tackled through a controlled release strategy in which retinal is continuously delivered to the skin via sustained release from proretinal nanoparticles. The water dispersible proretinal nanoparticles are stable when kept in water at neutral $\mathrm{pH}$ and at room temperature for 8 months under light-proof conditions, and show sustained release of retinal into human synthetic sebum at a $\mathrm{pH}$ of 5 . In the daily topical application tests performed for 4 weeks on rats' skin, the nanoparticles showed superior ability to increase epidermal thickness compared to RA and retinal, with no skin irritation observed for the proretinal particles, but severe skin irritation observed for RA and free retinal. When tested under occlusion conditions in human volunteers, insignificant skin irritation was observed for the proretinal nanoparticles. The 12-week, double-blind, split-face study on human volunteers indicates better antiaging efficacy of the particles as compared to the free RA.

Keywords: antiaging, sebum, release, drug delivery, skin

\section{Introduction}

Vitamin A (retinol) and its naturally occurring and synthetic derivatives such as retinaldehyde (retinal), retinoic acid (RA), retinyl palmitate, and retinyl acetate, collectively referred to as retinoids, are required for many critical biological processes. Since the skin is one of the principal sites for retinoid-dependent physiological effects, retinoids have been used as a prescription drug for the treatment of many skin disorders such as acne and rosacea. ${ }^{1,2}$ Retinoids display key regulatory functions in epidermal growth and differentiation. By binding to RA receptors and acting via retinoid X receptorsmediated transactivation or transrepression, ${ }^{3}$ they can increase the epidermal thickness and decrease wrinkling, roughness, and uneven melanin synthesis in the human skin. Retinoids are therefore potent bioactive agents that exhibit antiaging effects. ${ }^{4,5}$ Retinoic acid is considered a drug in many countries, available only by prescription, while retinol, retinal, and retinyl esters can be used in nonprescriptive cosmetic products. It is believed that any physiological effects caused by other retinoids on skin are the result of their conversion into RA. ${ }^{4}$ The interconversion of retinoids in cells occurs through a variety of metabolic processes, eg, retinol can be sequentially oxidized to retinal and RA in vivo. ${ }^{6,7}$ Nevertheless, it has been shown that retinol and retinal are capable of modulating gene expression in keratinocyte cell line HaCaT through two nuclear receptors directly with no need to be converted to the RA. ${ }^{8}$ Retinyl esters are regarded as a storage form of vitamin A, since they are precursors of all biologically active forms of vitamin A. ${ }^{9}$ The limitations on the enzymatic hydrolysis of the esters 
to retinol in the skin explain the decreased effectiveness of the ester derivatives of vitamin A, compared to retinol, retinal, and RA. ${ }^{6,10}$

Despite many beneficial effects, retinoids also possess a dose-related broad side effect profile. Skin irritations such as burning and stratum corneum peeling are the common side effects associated with the topical application of retinoids, ${ }^{11,12}$ and these side effects have long limited the use of retinoids despite their potent efficacy. Since the side effects are usually dose dependent, a strategy to supply retinoids to the skin at a steady amount, with minimal or no overdosing period, should help to lessen many side effects. For this reason, a controlled release system that can slowly and continuously supply the skin with undegraded retinoids should be an answer for the topical use of these compounds.

In addition to the side effects observed, retinal and other retinoids are also highly unstable products, mostly from autooxidation and photooxidation. ${ }^{13,14}$ All retinoids contain polyene substructure which is susceptible to oxidation. Oxidative cleavages can also occur via reactive oxygenated intermediates such as epoxides and peroxides, yielding various small carbonyl compounds. ${ }^{15-17}$ Dimerization and trans to cis configurational change at the double bonds of the polyene substructure have also been reported. ${ }^{18}$ Degradation of retinoids occurs not only in vitro but also in vivo. Ultraviolet B (UVB) and ultraviolet A (UVA) radiations can reduce the content of retinoids in the human epidermis. ${ }^{2,19,20}$ Reported attempts to improve retinoid stability include both chemical derivatization ${ }^{21}$ and physical encapsulation strategies. ${ }^{22-24}$ Examples of reported retinoid encapsulation systems include the use of solid lipid nanoparticles, ${ }^{25}$ formation of nanoparticles by complexation of RA with synthetic cationic polyelectrolytes such as polyethylene oxide-6-poly(L-lysine) and poly(ethylene oxide)-grafted-polyethyleneimine, ${ }^{26,27}$ complexation with synthetic polyamino acids, ${ }^{28}$ encapsulation into silica particles, ${ }^{29}$ and inclusion complexation with $\alpha$-cyclodextrin lipidic beads. ${ }^{30}$ Nevertheless, till now, no system offers a suitable stability for the cosmetic use of vitamin A derivatives, where a long shelf-life is typically required, and more importantly, none has demonstrated significant improvement in the dose-dependent side effects.

Here, we show that the proretinal nanoparticles (PRN), which can sustainably release retinal at skin $\mathrm{pH}$, can not only diminish normal skin irritation side effects but also maximize epidermal proliferation. This work includes chemical stability study of the prodrug nanoparticles and antiaging efficacy tests in both rats and human volunteers.

\section{Materials and methods \\ Preparation and chemical evaluation of PRN}

PRN were prepared by grafting retinal onto chitosan (CS, molecular weight of $\sim 40,000-50,000 \mathrm{Da}$, Taming Enterprise, Samut Sakhon, Thailand) polymer and allowing the retinylidene chitosan (RCS) to automatically self-assemble into nanoparticles. CS was allowed to react with retinal at the weight ratio of CS to retinal of 3:1. First, CS was dissolved in $0.1 \%$ acetic acid, and the $\mathrm{pH}$ of the obtained solution was adjusted to 5.9 using $\mathrm{NaOH}$. The final solution contained $45 \mathrm{mg}$ CS in $19.0 \mathrm{~mL}$ solution. The grafting reaction was started by slowly adding retinal ( $15 \mathrm{mg}$ in $1.0 \mathrm{~mL}$ of ethanol) dropwise into the two-neck round bottom flask containing aqueous $\mathrm{CS}$ suspension at $5^{\circ} \mathrm{C}$ in the dark, under ultrasonic (40 kHz) and $\mathrm{N}_{2}$ atmospheric conditions (Figure 1). The $\mathrm{pH}$ of the solution was slowly adjusted to 6.8 after 2 hours. Progress of the reaction was monitored by subjecting aliquots of the reaction mixture to FT-IR analysis. Dry product was obtained by freeze-drying the obtained aqueous suspension. Reactions performed at weight ratio of CS to retinal of 1:1
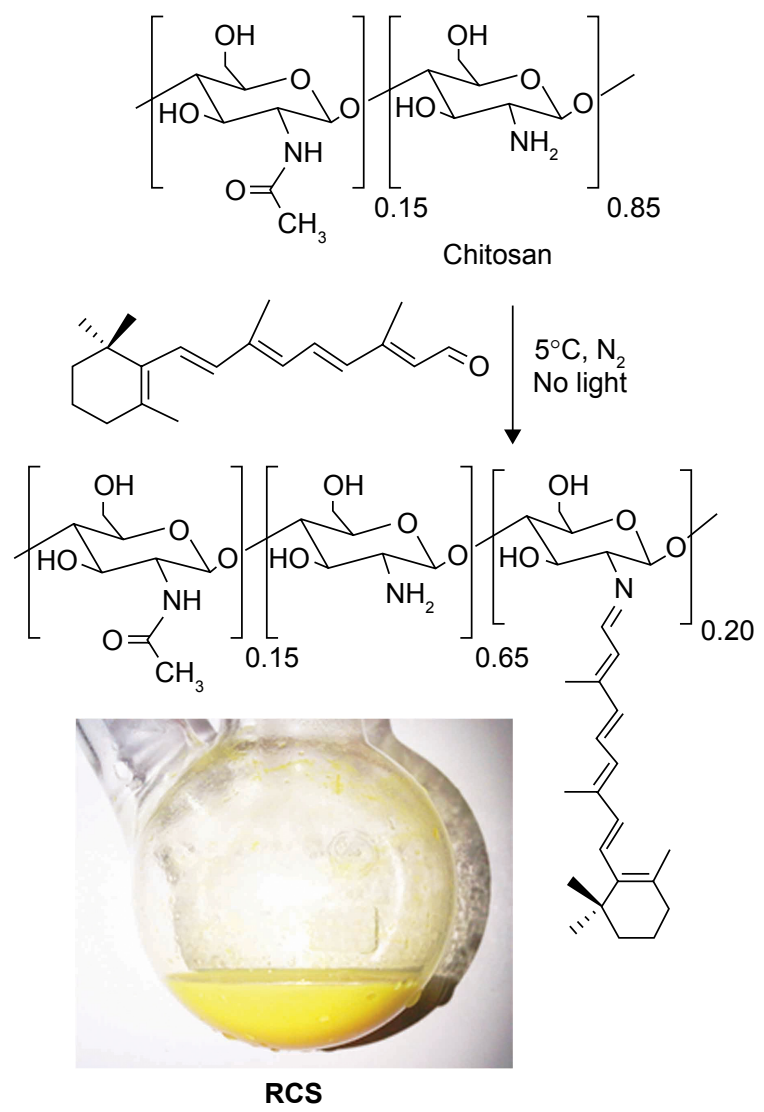

Figure I Synthesis of RCS and appearance of the self-assembled RCS aqueous suspension into PRN (bottom image).

Abbreviations: RCS, retinylidene chitosan; PRN, proretinal nanoparticles. 
and 2:1 were carried out similarly except that the weight of the retinal used was 45.0 and $22.5 \mathrm{mg}$, respectively.

RCS is a yellowish powder. The degree of retinal substitution in RCS is $0.20-0.22$. Attenuated total reflection Fourier transform infrared (ATR FT-IR) spectroscopy $\left(\mathrm{cm}^{-1}\right): 3,282$ ( $\mathrm{N}-\mathrm{H}$ str and $\mathrm{O}-\mathrm{H}$ str), 2,867 (C-H str), 1,620 (C=N str), 1,555 (amide II), 1,150 (C-O-C str), and 1,023 (C-O str).

Retinal loading of the PRN (water dispersible particles of CS grafted with retinal through imine linkage between amino functionality of the glucosamine units in the CS and aldehyde functionality of the retinal) was evaluated by stirring the PRN $(20 \mathrm{mg})$ in $0.1 \mathrm{M}$ aqueous $\mathrm{HCl}(5 \mathrm{~mL})$ for 30 minutes under $\mathrm{N}_{2}$ atmosphere to allow complete hydrolysis of the reversible covalent bonds that link retinal to the biopolymers, then partitioning the mixture with ethyl acetate $(2 \times 3 \mathrm{~mL})$, and quantifying the released retinal in the ethyl acetate fraction using ultraviolet-visible (UV-vis) spectrophotometry $\left(\lambda_{\max }\right.$ of $340 \mathrm{~nm}$ ) with the aid of the calibration curve. The extraction process was repeated until no retinal was detected. Confirmation of retinal was carried out by evaporating the extract under nitrogen and subjecting to ${ }^{1} \mathrm{H}$ nuclear magnetic resonance analysis.

\section{Morphology of PRN}

PRN was evaluated for its zeta potential in water by dynamic light scattering (DLS) analysis (Zetasizer nano series S4700; Malvern Instruments, Malvern, UK). The particles were also subjected to scanning electron microscopic (SEM, JSM-6400; JEOL, Tokyo, Japan) and transmission electron microscopic (TEM, JEM-2100; JEOL) analyses.

\section{Stability of PRN}

Stability of the retinoid in PRN was evaluated in comparison with free retinal in vitro under accelerated degradation and normally kept conditions. Aqueous suspension of PRN was diluted with water to obtain a final concentration of $0.317 \mathrm{mg} / \mathrm{mL}$ of retinal. The solution of free retinal (to obtain a final concentration of $0.317 \mathrm{mg} / \mathrm{mL}$ ) was prepared with the help of ethanol to solubilize the materials. For the stability test under accelerated condition, the samples (PRN suspension and retinal solution) were incubated for 5 hours at $40^{\circ} \mathrm{C}$ under direct sunlight (UVA and UVB with an intensity of $\sim 3-5$ and $\sim 0.10-0.15 \mathrm{~mW} / \mathrm{cm}^{2}$, respectively, measured using the UVA-400C and UVB-500C power meters; National Biological Corporation, Twinsburg, OH, USA) with continuous exposure to air through stirring at $300 \mathrm{rpm}$. The degradation of retinal was quantitatively monitored through a decrease in the absorbance of the $5 \mathrm{~mL}$ aliquots taken at $0,30,60$, and 120 minutes postincubation at $365 \mathrm{~nm}$ (for the retinylidene moieties) or $340 \mathrm{~nm}$ (for retinal). For the stability test under normally kept conditions, the samples were kept in light-proof conditions at $25^{\circ} \mathrm{C}$ for 8 months, and the degradation was monitored using procedures similar to those performed for the accelerated condition. The experiments were carried out in triplicate.

\section{Release of retinal into human synthetic sebum}

Human synthetic sebum was prepared as previously described by mixing together the following compounds: $1.240 \mathrm{~g}$ squalene, $2.500 \mathrm{~g}$ wax monoester (jojoba oil), $4.470 \mathrm{~g}$ triglyceride (triolein), $1.700 \mathrm{~g}$ fatty acid (oleic acid), and $50 \mathrm{mg}$ vitamin $\mathrm{E} .{ }^{31}$ In vitro release of retinal from PRN was measured by introducing $0.6 \mathrm{~mL}$ of aqueous suspension of PRN (the concentration of retinal was $3.33 \mu \mathrm{g} / \mathrm{mL}$ ) into $1.0 \mathrm{~mL}$ synthetic sebum. The $\mathrm{pH}$ of the particle suspension was adjusted to 5.0 just prior to the addition into the sebum. The mixture was stirred at $4^{\circ} \mathrm{C}$ in the dark for 30 minutes, $1,2,4$, and 8 hours. At required time, the mixture was centrifuged $(1,000$ rpm, 3 minutes) and the liquid was collected and subjected to UV-vis absorption spectrophotometry, and the amount of retinal was estimated through the use of a standard reference curve constructed using freshly prepared retinal standards.

\section{Skin irritation and histological changes of rat skin}

Male rats aged 10-12 weeks at the beginning of the experiments were obtained from the National Laboratory Animal Center (Mahidol University, Bangkok, Thailand). General health status and laboratory conditions were controlled according to standard operating procedures (temperature $25^{\circ} \mathrm{C} \pm 2{ }^{\circ} \mathrm{C}$, relative humidity $55 \% \pm 10 \%, 12$-hour light/dark cycle) with food and water available ad libitum. This study and the experimental procedures were approved by both the Ethics Committee and the Institutional Animal Care and Use Committee (IACUC) of Chulalongkorn University. The animal welfare conformed to the guidelines of the Care and Use of Laboratory Animals of the Thai National Animal Center. Rats were randomly divided into five groups, namely RA, retinal, PRN, water, and control groups, with five rats per group.

Rats in each group were treated once daily with the corresponding samples. Liquid sample $(200 \mu \mathrm{L})$ was applied evenly and massaged in a circular motion for 1 minute, using a micropipette with a disposable tip, to the dorsal skin of $4 \mathrm{~cm}^{2}$ area. The samples used were as follows: 1) $3.33 \mathrm{mM}$ $(0.1 \% \mathrm{w} / \mathrm{v}) \mathrm{RA}$ solution, 2) $3.33 \mathrm{mM}$ retinal solution, 
3) PRN suspension (at a concentration corresponding to $3.33 \mathrm{mM}$ retinoid), and 4) water. Rats in the control group received no sample application. Signs of irritation were evaluated by veterinarians. Tolerance was graded according to $0-3$ scale: $0=$ no irritation sign, $1=$ mild stinging and no objective signs, $2=$ stinging burning and visible erythema with subsequent fine scaling, and $3=$ frank burning and erythema with edema. ${ }^{32}$ The $\mathrm{pH}$ of skin after sample application was measured at 20 minutes post-sample application by pressing the wet $\mathrm{pH}$ paper onto the skin for 2 minutes and observing the $\mathrm{pH}$ value through the color change of the paper using a standard $\mathrm{pH}$ paper color calibration chart.

Hair at the tested area was shaved off under anesthesia, and then the full-thickness skin was sampled by $8 \mathrm{~mm}$ punch biopsy at days 0,7 , and 28 . The tissue samples were fixed in $10 \%$ buffered formalin, embedded in paraffin, sectioned at $4 \mu \mathrm{m}$ thickness (five sections per sample), stained with hematoxylin and eosin (H\&E), and then examined under light microscope. Thickness of the epidermis $(\mu \mathrm{m})$ from the base of the stratum corneum to the basement membrane assessed in ten high-power fields was measured randomly (five repetitions per slides), and then the mean and standard deviation (SD) per individual were calculated, after which the mean and SD per group were calculated.

\section{Single application closed patch test}

Sixteen healthy female and four healthy male volunteers aged 15-65 years with no underlying skin disease or skin lesion on the test area (upper back) were recruited (Table S1 for details of volunteers). All volunteers were nonpregnant and not breastfeeding. Volunteers receiving immune suppressive therapy, corticosteroids, antihistamines, and/or chemotherapy in the previous 2 weeks were excluded. Written informed consent was obtained from each participant. The same dermatologist performed the whole evaluation. Irritancy was scored by the using a grading scale of $0-4$ as follows: $0=$ no reaction; $\pm 0.5=$ doubtful reaction (glazed appearance of the site/barely perceptible erythema); $1=$ weak reaction (slight erythema or dryness across most of the treatment site); $2=$ moderate reaction (moderate erythema, possibly spreading with barely perceptible edema at the margin, papules may be present); $3=$ strong reaction (moderate erythema with generalized edema); $4=$ severe reaction (severe erythema with severe edema, with or without vesicle, pustule, and ulcer). ${ }^{33}$ Statistical analysis was done using McNemar's test and $P$-value of less than 0.005 was considered significant.

The test samples $(20 \mu \mathrm{L}$ ) which included PRN (at $3.33 \mu \mathrm{mol}$ retinal equivalent $/ \mathrm{mL}$ ), distilled water (negative control), and sodium dodecyl sulfate (SDS; $2.5 \% \mathrm{w} / \mathrm{v}$ solution, positive control) were applied as a patch on normal-appearing skin on the upper back of test subjects under occlusion with an $8 \mathrm{~mm}$ diameter Finn chamber (Epitest Ltd., Tuusula, Finland) for 24 hours. After the 24-hour skin contact period, the patch was removed and signs of skin irritation were observed at 1, 24, and 48 hours post-patch removal. The test area was kept dry until the last day of observation.

\section{Antiaging efficacy}

Hydrogel containing PRN was prepared by dispersing the PRN into the hydrogel $(0.75 \mathrm{~g}$ acrylates/C10-30 alkyl acrylate cross polymer, $0.22 \mathrm{~g}$ sodium hydroxide, $0.6 \mathrm{~g}$ phenoxyethanol, $0.3 \mathrm{~g}$ sodium benzoate, $98 \mathrm{~mL}$ water) to obtain $0.833 \mu \mathrm{mol}$ retinal equivalent $/ \mathrm{mL}$. Hydrogel containing free RA was used as control $(0.833 \mu \mathrm{mol} \mathrm{RA} / \mathrm{mL}$ or $0.025 \% \mathrm{w} / \mathrm{v} \mathrm{RA})$.

The study protocol was reviewed and approved by the Ethical Review Committee of the Faculty of Medicine, Ramathibodi Hospital, Mahidol University, and the experiments were conducted according to the Declaration of Helsinki principles. The antiaging efficacies of PRN and RA were compared using a 12-week-randomized, double-blind, split-face comparative study. The test was carried out on 30 healthy Thai female volunteers aged 30-62 years with no underlying skin disease or skin lesion on the test area (periorbital skin) (see Table S2 for details of volunteers). All volunteers were not pregnant, not breastfeeding, and had not used oral or topical vitamin A and vitamin A derivatives during the previous 12 weeks. All subjects agreed to use only basic moisturizing products for daily skin care during the study.

One side of the patient's periorbital skin $\left(\sim 15 \mathrm{~cm}^{2}\right)$ was randomly assigned to receive PRN hydrogel sample $(0.25 \mathrm{~mL}$ gel for each application, equivalent to $\sim 1.39 \times 10^{-5} \mathrm{mmol}$ retinoid $/ \mathrm{cm}^{2}$ ) and the other side was assigned to receive RA sample $(0.25 \mathrm{~mL}$ gel for each application, equivalent to $4.17 \mu \mathrm{g} / \mathrm{cm}^{2}$ or $1.39 \times 10^{-5} \mathrm{mmol}$ retinoid $/ \mathrm{cm}^{2}$ ), twice a day (morning and night, after showering) for 12 weeks.

The self-application of the sample was carried out by hand massaging the gel into the skin. Evaluation of the tested area was carried out prior to (T0), and periodically during (at 4 and 8 weeks post-first application) and at the end (12 weeks post-first application) of the treatment under a controlled temperature and humidity room (temperature: $22^{\circ} \mathrm{C} \pm 2{ }^{\circ} \mathrm{C}$, hygrometry: $50 \% \pm 10 \%$ ). The subjects rested quietly for at least 15 minutes in the controlled room prior to assessment. The photographs of the face were taken (facial position: left 
$37^{\circ}$, center $0^{\circ}$, right $37^{\circ}$ ) under the controlled parameters, eg, camera setting, camera positioning, and light conditions, using the VISIA Complexion Analysis System (Canfield Scientific Inc., Parsippany-Troy Hills, NJ, USA). Skin aging (fine lines and wrinkles) was evaluated using a Visioscan device and its dedicated software (Courage-Khazaka, Cologne, Germany). Seven skin parameters including surface, volume, energy, entropy, homogeneity, contrast, and variance values were acquired. ${ }^{34}$ Statistical analysis was conducted using SPSS statistics vision 17.0 software (SPSS Inc., Chicago, IL, USA), using a two-tailed paired Student's $t$-test (normality of distributions checked) or the Wilcoxon test (normality of the distributions rejected) at a significance threshold of $5 \%$. Verification of the normality of the distributions was carried out using Shapiro-Wilk test, at 1\% threshold.

\section{Results and discussion PRN}

FT-IR spectrum of the reaction mixtures obtained from the reactions at retinal to $\mathrm{CS}$ weight ratios of 1:3 showed a new absorption peak at $1,620 \mathrm{~cm}^{-1}(\mathrm{C}=\mathrm{N}$ stretching [str] $)$ and a complete disappearance of the peak at $1,709 \mathrm{~cm}^{-1}(\mathrm{C}=\mathrm{O}$ str of aldehyde) (Figures S1 and $\underline{\mathrm{S} 2}$ ). It should be noted here that FT-IR spectra of the reaction mixtures obtained from the reactions at retinal to $\mathrm{CS}$ weight ratios of 1:1, 1:2, and 1:3 (Figure S2) revealed a complete disappearance of the $\mathrm{C}=\mathrm{O}$ stretching vibration absorption at $1,709 \mathrm{~cm}^{-1}$ (indicating no left over retinal) for only the reaction performed at the 1:3 weight ratio. This implies that under our reaction conditions, all the retinal moieties were converted to imine linkages when the reaction was carried out at the retinal to CS weight ratio of $1: 3$. The $1: 3$ weight ratio corresponds to the mole ratio of glucosamine unit to retinal of 4.5:1. Therefore, not all of the amino groups on the CS were grafted and converted into retinylidene moieties. The obtained product appears as a yellowish suspension. The diluted suspension gives absorption spectrum with a maximum absorption at $350 \mathrm{~nm}$ (Figure S3). This indicates the presence of retinylidene moieties in the structure.

The presence of retinal in the freeze-dry product was confirmed through the regeneration of aldehyde functional group upon acid hydrolysis (observed through the appearance of absorption peak at $1,709 \mathrm{~cm}^{-1}$ in ATR FT-IR spectrum of the acid-treated product). The retinal loading of $34.7 \% \pm 1.3 \%$ $\mathrm{w} / \mathrm{w}$ in the freeze-dry product was determined by hydrolyzing the material with $0.1 \mathrm{M} \mathrm{HCl}$, partitioning the mixture with ethyl acetate, and quantifying the released retinal in the ethyl acetate fraction using UV-vis spectrophotometry with the aid of the calibration curve.
SEM and TEM images of the product (Figures 2 and $\underline{\text { S4}}$ ) reveal spherical particles with an average diameter of 240.1 $29 \mathrm{~nm}$ (determined from SEM images using ImageJ software; National Institutes of Health, Bethesda, MD, USA). Thus, we speculate that the RCS polymer chains probably selfassembled into spherical particles under the reaction conditions used. This is very likely since retinylidene moieties are hydrophobic and would try to be away from water medium by positioning themselves at the inside of the self-assembled particles. The particle size obtained from DLS is $373 \pm 12 \mathrm{~nm}$ with a polydispersity index of 0.302 (Figure S4), indicating some swelling of the particles in water, likely from the hydration of CS moieties. The zeta potential value of $29.7 \pm 0.2 \mathrm{mV}$ explains the minimum aggregation of the particles (when suspended in water) through the surface charge repulsion among particles. Thus, the RCS nanoparticles were successfully synthesized, and the obtained particles were called PRN. It should be noted here that the mechanism of hair follicle entrapment of particles involves the pushing of particles into follicles with hair shafts acting as sliding bars. ${ }^{35}$ Effective entrapment depends on particle size (not too small and not too large) and hair shaft movement, through skin massage, ultrasound treatment, or other strategies, is usually needed. ${ }^{36} \mathrm{It}$ has been shown that the particle sizes of 200-400 nm provide a good range for human hair follicle entrapment. ${ }^{35}$

\section{Stability of PRN}

Under the accelerated condition $\left(40^{\circ} \mathrm{C}\right.$, water, light, and $\mathrm{O}_{2}$ exposure), the relative retinoid degradation rates for free retinal and PRN during the first hour were 1 and 0.2, respectively (Figure 3). When kept as an aqueous suspension at $25^{\circ} \mathrm{C}$ under a light-proof condition for 8 months, PRN showed less than $10 \%$ deterioration of the retinoid, whereas the free retinal degradation was $>90 \%$. This indicates excellent chemical stability of the retinoid in the PRN.

As mentioned earlier, the hydrophobic retinylidene moieties probably localize themselves inside the PRN to avoid contact with water. This phase separation of the grafted retinylidene

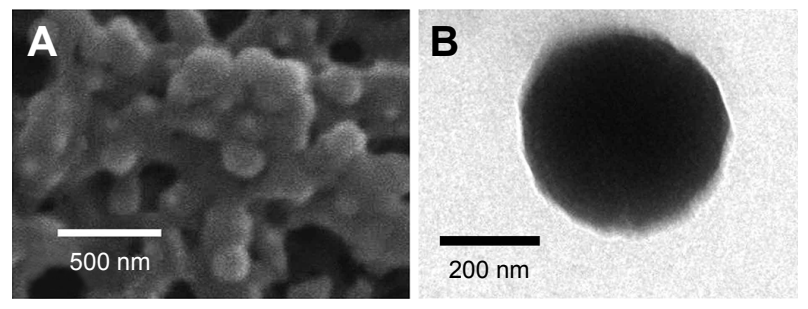

Figure 2 SEM (A) and TEM (B) images of PRN.

Abbreviations: SEM, scanning electron microscope; TEM, transmission electron microscope; PRN, proretinal nanoparticles. 


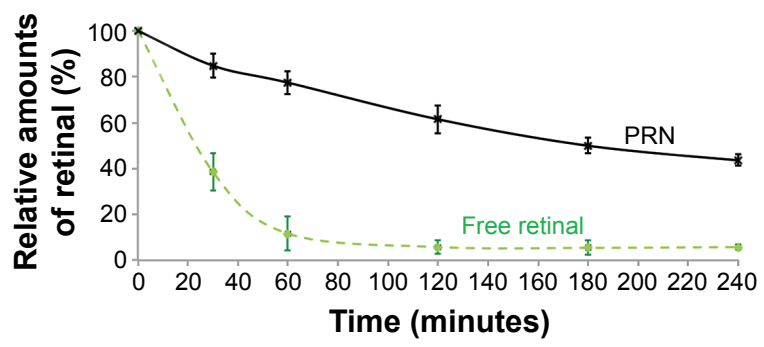

Figure 3 Plots showing the stability of PRN when kept as aqueous suspension under an accelerated condition as compared with free retinal.

Note: Accelerated condition: $40^{\circ} \mathrm{C}$ under sunlight (UVA and UVB with an intensity of $\sim 3-5$ and $\sim 0.10-0.15 \mathrm{~mW} / \mathrm{cm}^{2}$, respectively) and continuous air exposure. Abbreviations: PRN, proretinal nanoparticles; UVA, ultraviolet A; UVB, ultraviolet $B$.

moieties from the water medium helps stabilize the imine bonds of the PRN. With the hydrophobic environment at the interior of PRN, attack by water-soluble free radicals is not easy. Aggregation into particles also reduces the exposure of retinylidene moieties to light. These arrangements lead to the higher stability of the retinoid in the PRN over the free retinal.

\section{Release of retinal into synthetic sebum}

The sustained release of retinal from PRN into human synthetic sebum at $\mathrm{pH} 5$ was observed in vitro (Figure 4). The release of retinal from PRN was faster at the beginning, followed with a slower release at a later time and completed in $\sim 8$ hours. Under similar conditions but at $\mathrm{pH} 7.0$, insignificant amount of retinal release was detected from the PRN at 8 hours. It should be noted here that at the same retinoid concentration, free retinal dissolves quickly in the sebum; thus, the release of free retinal into the sebum can be considered instant.

This sustained release character at skin $\mathrm{pH}$ should minimize the irritation side effects of retinal. Our hypothesis is

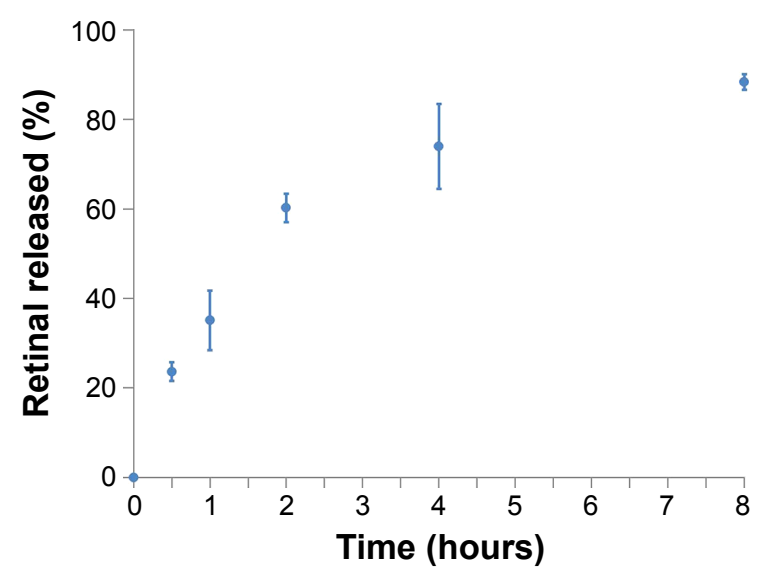

Figure $4 \mathrm{~A}$ plot showing the release of retinal from PRN into the human synthetic sebum at $\mathrm{pH}$ 5.0.

Abbreviation: PRN, proretinal nanoparticles. that if the concentration of retinal delivered into the skin from a single application can be spread out during the day, the dose-related irritation side effects should be diminished, whereas the antiaging effect should be maximized. To find out, we set up the in vivo experiment in which both the skin irritation and the ability to increase epidermal thickness by the free retinal and RA were compared with those of the PRN.

\section{Tolerance profiles and histopathology in skin of rats}

At the end of the 4-week daily topical treatment of samples (RA, retinal, PRN, and distilled water) on the dorsal skin of rats in corresponding groups (each application provided the covering of $0.1665 \mu \mathrm{mol} \mathrm{retinoid} / \mathrm{cm}^{2}$ or $\sim 0.05 \mathrm{mg}$ retinoid $/ \mathrm{cm}^{2}$ ), we clearly observed irritation of grades $2-3$ on all five rats of the RA- and retinal-treated groups. The rest of the rats (PRN- and distilled water-treated groups) showed no skin irritation (grade 0 ) at the treated area. Retinoic acidtreated group showed a more severe burning than the retinaltreated group (Figure 5 and Table S3). The irritation effects of RA and retinal agree well with the previous reports. ${ }^{11,12}$

The epidermal thickness at the interfollicular areas of the biopsied rat skins was histologically measured and the results showed a significant increase in the epidermal thickness in the three retinoid-treated groups (RA, retinal, and PRN) compared to that in the control (water-treated group) (Figure 6). At the same retinoid dosage, PRN produced a significantly higher and faster increase of epidermal thickness than retinal and RA (Figure 6). The results observed for RA and retinal agree well with antiaging effects of the materials. ${ }^{2-4}$ The better result of PRN can be explained through the sustained release of retinal from PRN (Figure 4), which can probably supply the skin tissue with effective retinal concentration for 8 hours after each daily application. It should be noted here that the biopsied skins of all groups showed similar thickness of the dermis. However, we noticed that the dermis of the skins from the RA and retinal groups was inflamed with obvious fibrous tissue, whereas the dermis of the skins from the PRN group showed no inflammation and fibrous tissue.

The $\mathrm{pH}$ of the rat skin is normally $\sim 5-6,{ }^{37}$ and we observed that the skin $\mathrm{pH}$ did not change upon massage with the samples. Therefore, when the samples were massaged into the rat skin, they were actually exposed to the $\mathrm{pH} 5-6$ environment, and the sustained release of retinal from PRN similar to that observed in the in vitro release experiment (at $\mathrm{pH}$ 5) probably took place. The sustained release of retinal prevented the overdose of retinal in the skin tissue immediately after the application, thus solving the irritation 

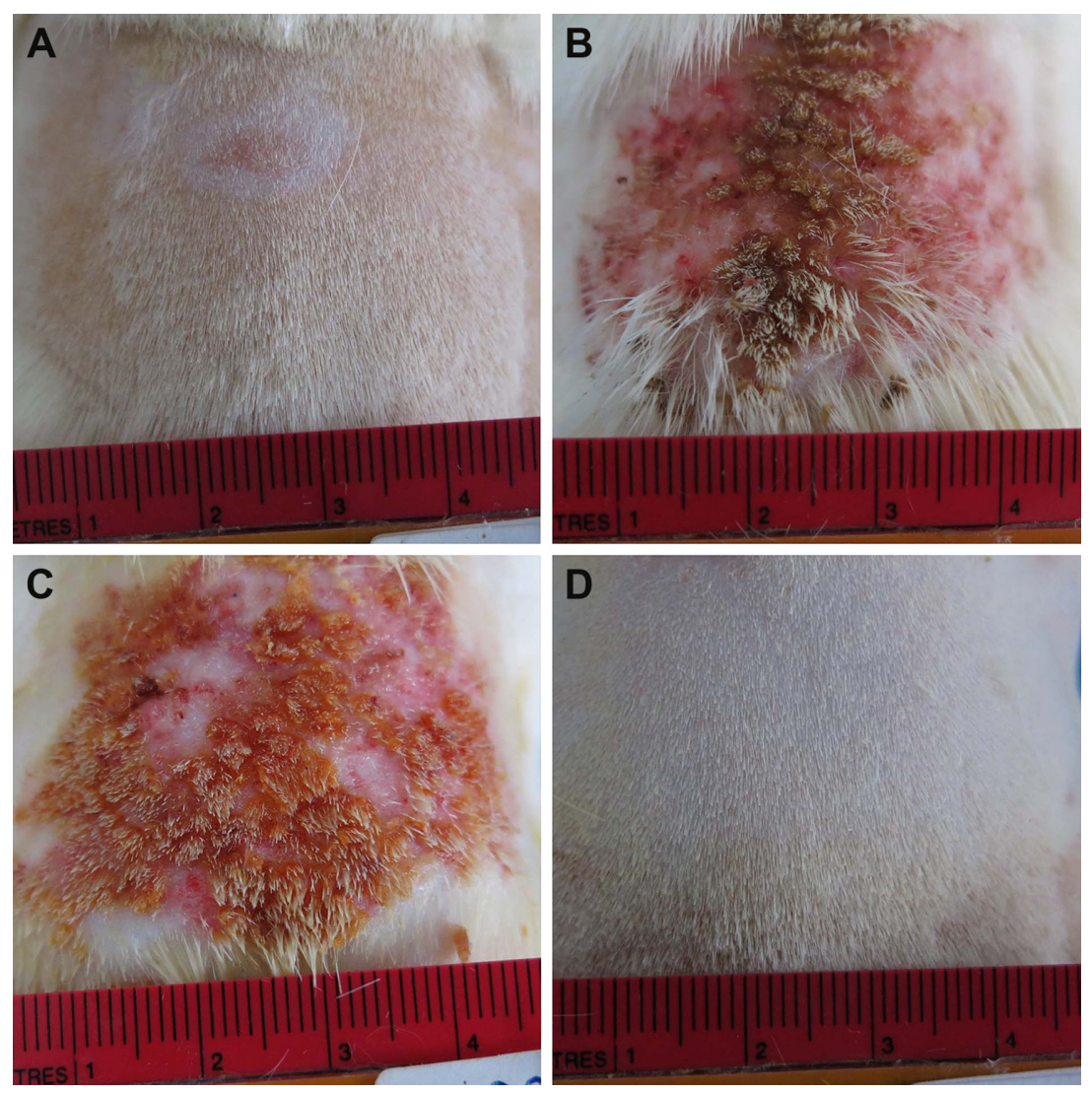

Figure 5 Photographic images of the skin at 14 days after daily treatment.

Notes: Rat skin at I 4 days after the daily treatment of $4 \mathrm{~cm}^{2}$ dorsal skin of rats daily with $200 \mu \mathrm{L}$ of (A) distilled water, (B) $0.1 \% \mathrm{w} / \mathrm{v} \mathrm{RA} \mathrm{or} 0.0033 \mathrm{mmol} / \mathrm{mL}$, (C) $0.0033 \mathrm{mmol} / \mathrm{mL}$ retinal, and (D) PRN (at the concentration corresponding to $0.0033 \mathrm{mmol} / \mathrm{mL}$ of retinal).

Abbreviations: PRN, proretinal nanoparticles; RA, retinoic acid.

problem. In contrast, the skin tissues receiving the retinal and RA dosage all at once immediately after each application exhibited skin irritation responses. The quickly depleted retinal and RA concentrations also resulted in less epidermal thickening activity compared to PRN where longer effective drug concentration could probably be maintained in the skin tissue.

With nonirritating character under nonocclusion condition and excellent epidermal thickening stimulation results observed in rat skin, we next evaluated the irritation potential
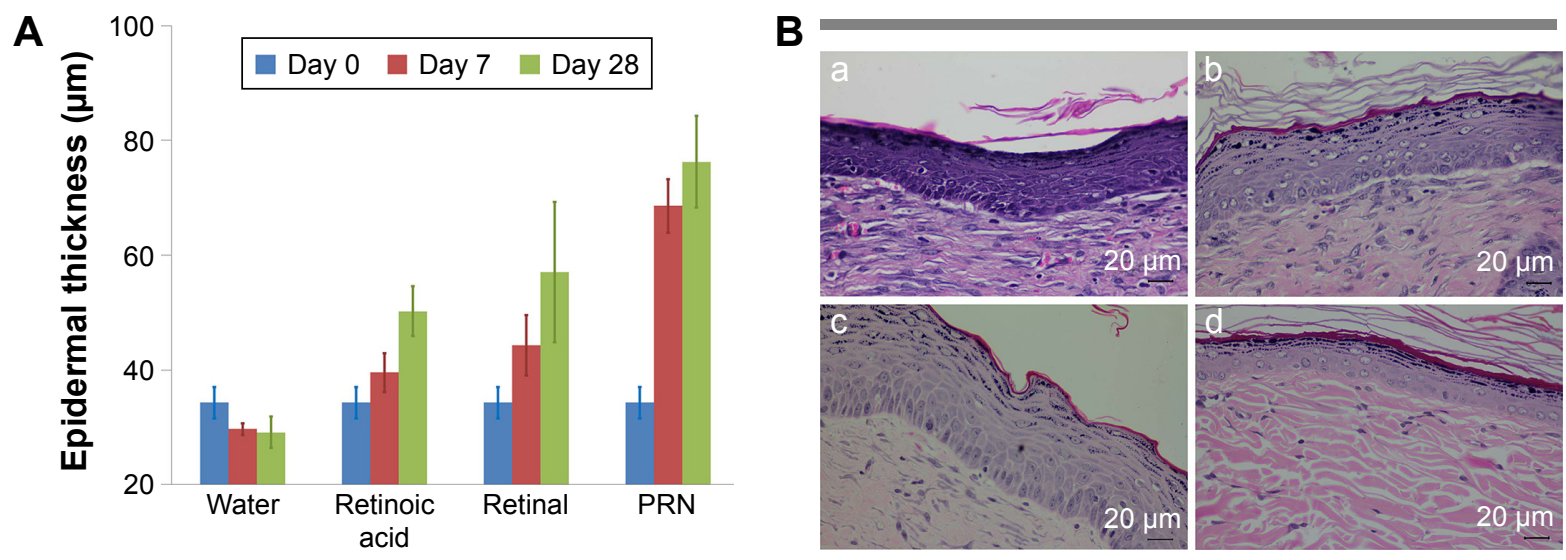

Figure 6 Epidermal thicknesses.

Notes: (A) A plot showing average epidermal thicknesses at various treatment times. (B) Representative pictures of the H\&E-stained tissue sections after 28 days of treatment with (a) $0.1 \% \mathrm{w} / \mathrm{v}$ or $0.0033 \mathrm{mmol} / \mathrm{mL}$ RA, (b) $0.0033 \mathrm{mmol} / \mathrm{mL}$ retinal, (c) PRN (at $0.0033 \mathrm{mmol} / \mathrm{mL}$ retinal equivalent), and (d) distilled water. Abbreviations: H\&E, hematoxylin and eosin; PRN, proretinal nanoparticles; RA, retinoic acid. 
of PRN in 20 healthy human volunteers under occlusion condition.

\section{Irritation potential of PRN in volunteers under occlusion condition}

The retinoid concentration in the aqueous PRN suspension used in the study was $0.0033 \mathrm{mmol} / \mathrm{mL}$ (equivalent to $\sim 0.1 \% \mathrm{w} / \mathrm{v}$ retinal). This high retinoid concentration is used in some commercial formulations in which irritation responses have been reported. In this study, the test was carried out at a final retinoid dose of $\sim 0.01 \mathrm{mg} / \mathrm{cm}^{2}$ on the skin, a typical dose equivalent to the $0.1 \% \mathrm{w} / \mathrm{v}$ retinoid formulation. All subjects participated until the study was completed. The irritation responses observed for PRN were not significantly different from those observed for distilled water but were significantly different from those observed for $2.5 \%$ SDS (Table S4). The $2.5 \%$ SDS solution caused a cutaneous irritant response in eleven subjects (55\%) at 1 hour, 17 subjects $(85 \%)$ at 24 hours, and 16 subjects $(80 \%)$ at 48 hours post-opening of the patch, with the mean severity grades of $0.82 \pm 0.60,1.06 \pm 0.66$, and $1.00 \pm 0.63$, respectively. PRN showed doubtful reactions (grade 0.5 ) in only one subject at 1 hour post-opening of the patch and the reaction completely disappeared at 24 hours post-opening of the patch. The analysis of variance using McNemar's test showed that the irritation potential of PRN was not significantly different from that of distilled water but was significantly different from that of $2.5 \%$ SDS (Table S5). The results indicated insignificant irritation character of the PRN.

With good chemical stability, well-sustained release at skin $\mathrm{pH}$, excellent epidermal thickening stimulation effect in rat, and insignificant skin irritation in human, PRN is a promising retinoid. The question, however, remains whether this PRN can produce significant antiaging effect on the human skin. The 12-week-randomized, double-blind, split-face study in 30 healthy human volunteers was, therefore, conducted to compare the antiaging efficacy between PRN and standard RA.

\section{Antiaging efficacy}

In this study, the antiaging effect was evaluated by monitoring various surface and texture parameters of the tested skin area. The PRN used had a retinoid concentration of $0.825 \mu \mathrm{mol} / \mathrm{mL}$ (equivalent to $\sim 0.025 \% \mathrm{w} / \mathrm{v}$ retinoid) and retinoid dose on the skin was $\sim 0.004 \mathrm{mg} / \mathrm{cm}^{2}$, a typical dose when the $0.025 \% \mathrm{w} / \mathrm{v}$ retinoid formulation was applied normally. Both PRN and RA produced significant overall antiaging effects on the tested skin area; however, better antiaging efficacy for the former over the latter was observed (Figure 7A, Table S6).
For the wrinkle-related parameters, PRN treatment showed more decrease in the surface parameter (higher surface area means higher numbers and bigger size of wrinkles) and volume parameter (virtual volume of liquid $\left[\mathrm{mm}^{3}\right]$ required to fill the wrinkle depth and higher volume parameter corresponds to higher numbers of wrinkles and deeper wrinkles) than RA treatment. Twofold decrease in the surface values was observed in the skins treated with PRN (-12.5\%) compared to the skins treated with RA (-7.1\%). PRN produced almost double statistically significant decreases in the volume parameters than the RA, ie, $-9.2 \%$ versus $-5.1 \%$ (Figure 7B, Table S6). For the skin color and texture-related parameters, the PRN treatment exhibited better improvement in homogeneity ( similarity of skin tone and colors of neighboring pixels, $+4.6 \%$ for PRN-treated side and $+2.9 \%$ for the RA-treated side) and more decrease in both contrast (contrast in skin colors of neighboring pixels, $-26.5 \%$ and $-20.2 \%$ for the PRN- and RA-treated sides, respectively) and variance (variation in skin colors of neighboring pixels, $-13.5 \%$ and $-9.1 \%$ for the PRN- and RA-treated sides, respectively) than the RA treatment (Figure 7C, Table S6).

The results indicate that the sustained release of retinal from the PRN not only solves the common skin irritation problem associated with retinoids but also produces effective antiaging effect on the human skin. This scenario is possible because the beneficial concentration of retinal in the skin tissue is lower than the concentration that causes skin irritation responses, and PRN can sustainably supply the tissue with the beneficial retinal concentration without overdosing the tissue with too high concentrations that will trigger skin irritation.

\section{Conclusion}

We herein report that by controlling the release of retinal, we can not only solve the problem of dose-related skin irritation side effects but can also maximize the stimulation of epidermal proliferation of the retinal. We show here that the chemically stable PRN can sustainably release retinal at skin $\mathrm{pH}$. The proretinal particles can be kept in water at $25^{\circ} \mathrm{C}$ under lightproof conditions for 8 months with less than $10 \%$ degradation. The proretinal particles show sustained release of retinal into human synthetic sebum at $\mathrm{pH}$ 5.0. The PRN produce no signs of skin irritation when applied daily onto the dorsal skin of rats at a dose of $0.05 \mathrm{mg}$ retinal equivalent $/ \mathrm{cm}^{2}$ for 4 weeks, whereas at the same dose, both retinal and RA cause severe skin irritation. At the same retinoid dosage, epidermal proliferation of rats' skins treated with PRN was significantly faster and more pronounced (approximately double) than in rats treated 
A
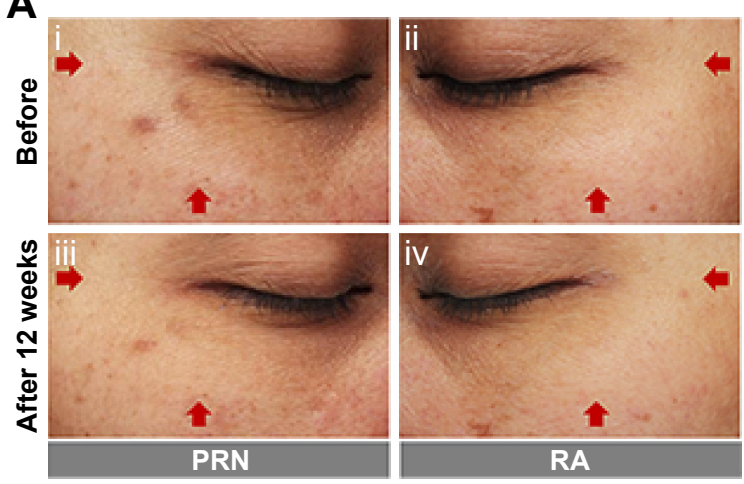

B

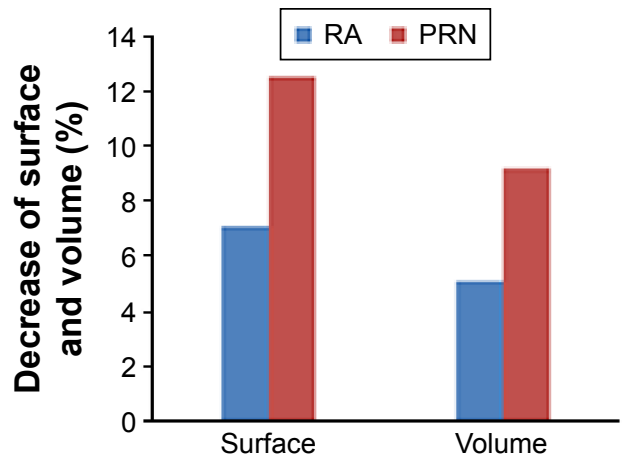

C

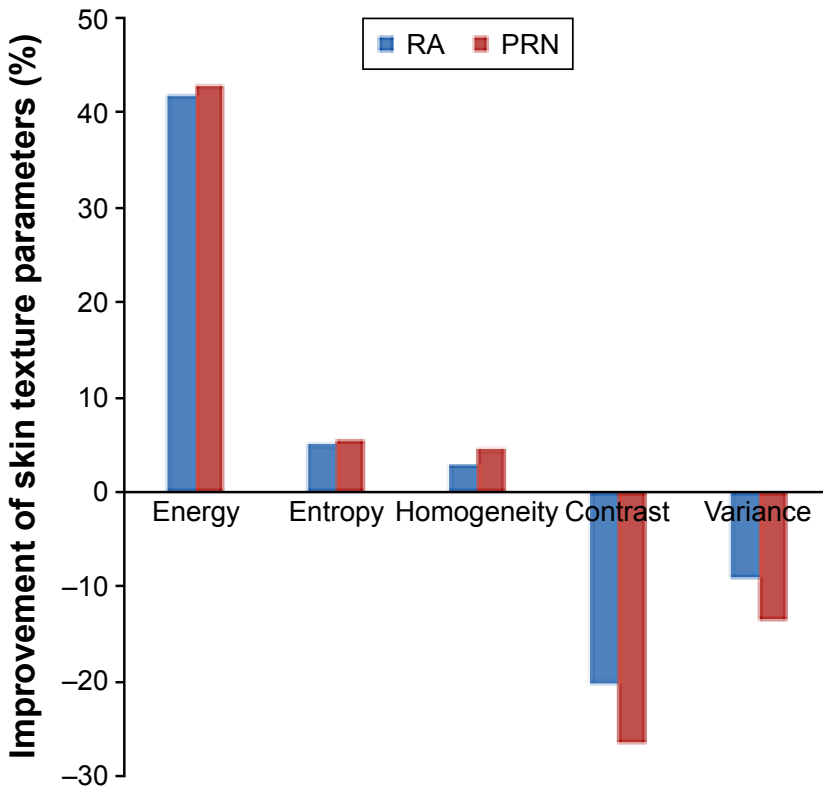

Figure 7 Antiaging efficacy of PRN and RA.

Notes: (A) Representative pictures of a preorbital skin area (tested area) of a volunteer at day 0 (before, i and ii) and day 84 (after 12 weeks post-first application, iii, iv) of the treatment (twice-daily application, at $\sim 0.004 \mathrm{mg}$ retinoid $/ \mathrm{cm}^{2}$ of the skin, for each application) with PRN (i and iii) and RA (ii and iv). Red arrows indicate areas with significant improvement after a 12-week treatment. (B) Decrease (\%) in the surface and volume parameters of volunteers' skin after the twice-daily applications of PRN and RA for 12 weeks. (C) Change (\%) in the skin texture parameters (energy, entropy, homogeneity, contrast, and variance) of volunteers after the twice-daily applications of PRN and RA for 12 weeks.

Abbreviations: RA, retinoic acid; PRN, proretinal nanoparticles.

with standard retinal or standard RA. Skin irritation evaluation in human volunteers under occlusion condition at a dose of $0.01 \mathrm{mg}$ retinal equivalent $/ \mathrm{cm}^{2}$ also indicates insignificant irritation of the PRN. Finally, tests in human volunteers at the twice-daily dose of $0.004 \mathrm{mg}$ retinoid $/ \mathrm{cm}^{2}$ clearly revealed better antiaging effects of PRN over RA.

\section{Acknowledgments}

The authors thank the Thailand Research Fund (RDG5850024), the 90th Anniversary of Chulalongkorn University (CU) Fund, the Ratchadapiseksompot Fund (GCRS58032301) from CU, and Nanotec-CU Center of Excellence on Food and Agriculture, Thailand.

\section{Disclosure}

The authors report no conflicts of interest in this work.

\section{References}

1. Varani J, Warner RL, Gharaee-Kermani M, et al. Vitamin A antagonizes decreased cell growth and elevated collagen-degrading matrix metalloproteinases and stimulates collagen accumulation in naturally aged human skin. J Invest Dermatol. 2000;114:480-486.
2. Didierjean L, Tran C, Sorg O, Saurat JH. Biological activities of topical retinaldehyde. Dermatology. 1999;199(Suppl 1):19-24.

3. Lefebvre P, Martin PJ, Flajollet S, Dedieu S, Billaut X, Lefebvre B. Transcriptional activities of retinoic acid receptors. Vitam Horm. 2005; 70:199-264.

4. Lupo MP. Antioxidants and vitamins in cosmetics. Clin Dermatol. 2001; 19:467-473.

5. Rafal ES, Griffiths CEM, Ditre CM, et al. Topical tretinoin (retinoic acid) treatment for liver spots associated with photodamage. $N$ Engl J Med. 1992;326:368-374.

6. Boerman MHEM, Napoli JL. Cellular retinol-binding protein-supported retinoic acid synthesis relative roles of microsomes and cytosol. $J$ Biol Chem. 1996;271:5610-5616.

7. Roos TC, Jugert FK, Merk HF, Bickers DR. Retinoid metabolism in the skin. Pharmacol Rev. 1998;50:315-333.

8. Amann PM, Schadendorf D, Owen RW, Korn B, Eichmüller SB, Bazhin AV. Retinal and retinol are potential regulators of gene expression in the keratinocyte cell line HaCaT. Exp Dermatol. 2011;20: 373-375.

9. Napoli JL. Retinoic acid biosynthesis and metabolism. FASEB J. 1996;10:993-1001.

10. Connor MJ. Oxidation of retinol to retinoic acid as a requirement for biological activity in mouse epidermis. Cancer Res. 1988;48: 7038-7040.

11. Kang S, Duell EA, Fisher GJ, et al. Application of retinol to human skin in vivo induces epidermal hyperplasia and cellular retinoid binding proteins characteristic of retinoic acid but without measurable retinoic acid levels or irritation. J Invest Dermatol. 1995;105: 549-556. 
12. Kim BH, Lee YS, Kang KS. The mechanism of retinol-induced irritation and its application to anti-irritant development. Toxicol Lett. 2003; 146:65-73.

13. Xia Q, Yin JJ, Cherng SH, et al. UVA photoirradiation of retinyl palmitate - formation of singlet oxygen and superoxide, and their role in induction of lipid peroxidation. Toxicol Lett. 2006;163:30-43.

14. Gaspar LR, Campos PMBGM. Photostability and efficacy studies of topical formulations containing UV-filters combination and vitamins A, C and E. Int J Pharm. 2007;343:181-189.

15. Karrer P, Hess W. Über die katalytische Oxydation von Vitamin A mit Sauerstoff und Platin zu Vitamin-A-aldehyd (eine neue Methode) [Via the catalytic oxidation of vitamin A with oxygen and platinum to vitamin A aldehyde (a new method)]. Helv Chim Acta. 1957;40:265-266. German.

16. Suyama K, Yeow T, Nakai S. Vitamin A oxidation products responsible for haylike flavor production in nonfat dry milk. J Agric Food Chem. 1983;31:22-26.

17. Isoe S, Hyeon SB, Katsumura S, Sakan T. Photo-oxygenation of carotenoids II. The absolute configuration of loliolide and dihydroactinidiolide. Tetrahedron Lett. 1972;13:2517-2520.

18. Mousseron-Canet M. Photochemical transformation of vitamin A. In: McCormick DB, Wright LD, editors. Methods in Enzymology XVIII. Part C. New York: Academic Press; 1979:591-615.

19. Creidi P, Vienne MP, Ochonisky S, et al. Profilometric evaluation of photodamage after topical retinaldehyde and retinoic acid treatment. J Am Acad Dermatol. 1998;39:960-965.

20. Sorg O, Tran C, Carraux P, Didierjean L, Saurat JH. Retinol and retinyl ester epidermal pools are not identically sensitive to UVB irradiation and anti-oxidant protective effect. Dermatology. 1999;199: 302-307.

21. Kim H, Kim B, Kim H, et al. Synthesis and in vitro biological activity of retinyl retinoate, a novel hybrid retinoid derivative. Bioorg Med Chem. 2008;16:6387-6393.

22. Hwang SR, Lim SJ, Park JS, Kim CK. Phospholipid-based microemulsion formulation of all-trans-retinoic acid for parenteral administration. Int J Pharm. 2004;276:175-183.

23. Kim DG, Jeong YI, Choi C, et al. Retinol-encapsulated low molecular water-soluble chitosan nanoparticles. Int J Pharm. 2006;319: 130-138.

24. Arayachukeat S, Wanichwecharungruang SP, Tree-Udom T. Retinyl acetate-loaded nanoparticles: dermal penetration and release of the retinyl acetate. Int J Pharm. 2011;404:281-288.
25. Jenning V, Schäfer-Korting M, Gohla S. Vitamin A-loaded solid lipid nanoparticles for topical use: drug release properties. J Control Release. 2000;66:115-126.

26. Thünemann AF, Beyermann J. Polyethylenimine complexes with retinoic acid: structure, release profiles, and nanoparticles. Macromolecules. 2000;33:6878-6885.

27. Bronich TK, Nehls A, Eisenberg A, Kabanov VA, Kabanov AV. Novel drug delivery systems based on the complexes of block ionomers and surfactants of opposite charge. Colloid Surf B. 1999;16:243-251.

28. Thünemann AF, Beyermann J, Von Ferber C, Löwen H. Immobilization of retinoic acid by polyamino acids: lamellar-structured nanoparticles. Langmuir. 2000;16:850-857.

29. Hwang YJ, Oh C, Oh SG. Controlled release of retinol from silica particles prepared in $\mathrm{O} / \mathrm{W} / \mathrm{O}$ emulsion: the effects of surfactants and polymers. J Control Release. 2005;106:339-349.

30. Trichard L, Fattal E, Besnard M, Bochot A. $\alpha$-Cyclodextrin/oil beads as a new carrier for improving the oral bioavailability of lipophilic drugs. J Control Release. 2007;122:47-53.

31. Wertz PW. Human synthetic sebum formulation and stability under conditions of use and storage. Int J Cosmet Sci. 2009;31:21-25.

32. Saurat JH, Didierjean L, Masgrau E, et al. Topical retinaldehyde on human skin: biological effects and tolerance. J Invest Dermatol. 1994; 103:770-774.

33. Wattanakrai P, Suwanachote S, Kulkollakarn S, Rajatanavin N. The study of human skin irritation of a novel herbal skin care product and ingredients by human single closed patch testing. J Med Assoc Thai. 2007; 90:1116-1122.

34. Ali A, Akhtar N, Chowdhary F. Enhancement of human skin facial revitalization by moringa leaf extract cream. Derm Alergol. 2014;31: $71-76$.

35. Lademann J, Richter H, Schanzer S, et al. Penetration and storage of particles in human skin: perspectives and safety aspects. Eur J Pharm Biopharm. 2011;77:465-468.

36. Paithankar D, Hwang BH, Munavalli G, et al. Ultrasonic delivery of silica-gold nanoshells for photothermolysis of sebaceous glands in humans: nanotechnology from the bench to clinic. J Control Release. 2015;206:30-36.

37. Gołyński M, Szczepanik M, Lutnicki K, et al. Biophysical parameters of rats' skin after the administration of methimazole. Bull Vet Inst Pulawy. 2014;58:315-319.
International Journal of Nanomedicine

\section{Publish your work in this journal}

The International Journal of Nanomedicine is an international, peerreviewed journal focusing on the application of nanotechnology in diagnostics, therapeutics, and drug delivery systems throughout the biomedical field. This journal is indexed on PubMed Central, MedLine, CAS, SciSearch $\AA$, Current Contents ${ }^{\circledR} /$ Clinical Medicine,

\section{Dovepress}

Journal Citation Reports/Science Edition, EMBase, Scopus and the Elsevier Bibliographic databases. The manuscript management system is completely online and includes a very quick and fair peer-review system, which is all easy to use. Visit http://www.dovepress.com/ testimonials.php to read real quotes from published authors. 